\title{
Lattice Boltzmann simulations of diffusion through native and steam-exploded softwood bordered pits
}

\author{
Patric Kvist ${ }^{1,3,4}$ - Adam Therning ${ }^{1}$ Tobias Gebäck ${ }^{2,4}$. \\ Anders Rasmuson ${ }^{1,3}$
}

Received: 13 January 2017/Published online: 19 July 2017

(C) The Author(s) 2017. This article is an open access publication

\begin{abstract}
Bordered pits connect adjacent tracheid cells in softwoods and enable water transport between them. Knowledge of how large molecules, such as polysaccharides and enzymes, are transported through pits is important to understand the extraction process of valuable biopolymers from wood. The main mass transport mechanism for large dissolved molecules in wood is diffusion, and this is investigated through mathematical modeling in the lattice Boltzmann framework utilizing SEM images and 3D reconstruction of an actual bordered pit to compute an effective diffusion coefficient. Confocal laser scanning microscopy is used to find the unobstructed diffusion coefficients in a free aqueous solution using fluorescent diffusion probes of dextran. The effect of steam explosion on pit structure is explored through the use of a simplified model. The importance of different components of a bordered pit is investigated using simulation data, and results show that the most important structural features are the borders. Expressions for the effective diffusion coefficient as a function of the free diffusion coefficient are presented for a native and for a steam-exploded pit, respectively.
\end{abstract}

Anders Rasmuson

rasmuson@chalmers.se

1 Department of Chemistry and Chemical Engineering, Chalmers University of Technology, Gothenburg, Sweden

2 Department of Mathematical Sciences, Chalmers University of Technology and University of Gothenburg, Gothenburg, Sweden

3 Wallenberg Wood Science Center, Stockholm, Sweden

4 SuMo Biomaterials, Gothenburg, Sweden 


\section{Introduction}

Climate change is a major contemporary environmental concern, and it is likely due to increased atmospheric concentration of greenhouse gases caused by the human use of fossil fuels (IPCC 2007). This has strengthened the incentive to consider alternative and more sustainable raw materials for the production of chemicals and value-added materials. A promising alternative is lignocellulosic biomass (FitzPatrick et al. 2010), especially from wood, as forests provide one of the largest sources of raw material for biorefining (Asikainen 2010). This study focuses on softwoods, such as spruce and pine, which are common in the northern hemisphere (Galbe and Zacchi 2002).

Softwood is a porous material that consists of longitudinal cells, tracheids, which are interconnected through openings referred to as pits. The main function of pits in a tree is to enable water conduction between tracheids. Three basic types of pit pairs exist; the simple, half-bordered, and bordered pair. Of the three pit pairs, the bordered pair has the most significance in softwood because softwood tissue is almost only prosenchyma cells, such as longitudinal tracheids (Siau 1984). A bordered pit consists of a permeable margo with a centered impermeable torus and a cell wall that overarches the margo and part of the torus (Sjöström 1993). Thus, understanding the porous structure and pit connections is important when it comes to impregnation and the processability of wood. Pits are considered as bottlenecks in mass transfer due to their small dimensions.

A cell wall is built up of a complex network of polysaccharides, cellulose, and hemicellulose and the irregular structured polymer lignin. In a material-driven biorefinery, separation of these three polymers with high molecular weight into separate streams is desired; however, the entangled structure of the cell wall aggravates such separation (Siau 1984). One way to increase yield is to use enzymes for the specific cleavage of certain bonds; however, for large enzymes to reach reaction sites in a cell wall, it is necessary to open the cell wall structure by pretreating the wood (Azhar et al. 2011). Steam explosion is the most common physicochemical pretreatment method for wood (Alvira et al. 2010) and has been studied in regard to enzyme accessibility (Jedvert et al. 2012; Muzamal et al. 2016) as well as structural effects both experimentally and by modeling (Muzamal et al. 2014, 2015). Wood porosity has also been shown to increase due to the expansion of water vapor during the release of high pressure to atmospheric pressure, which causes cracks in the cell wall and pits (Zhang and Cai 2006; Muzamal et al. 2015), thus increasing the mass transport rates through the cellular structure. Fractures and cracks in the pit structure in poplar wood have also been shown with a new technique, similar to steam explosion, called micro-explosion (Ma et al. 2016) where high pressure air is used instead of steam.

Due to the small scale of the structure of the pit, experimental measurements are extremely hard to perform to get accurate data for a single pit regarding flow or diffusion. Stamm (1946) derived a model for diffusion through a cellular structure analogous to electrical conductance with resistance in series according to various wood structures, such as lumens, cell walls, and the interior of the pit. Further 
development of said model of gases in dry wood to investigate the diffusion coefficient of bordered pits based on new data for structure and permeability in conifer wood was made by Petty (1973). Estimates of the resistance due to structural features were made based on the model, and it was found that the resistance to diffusion was as high as $15 \%$ for the pit membrane while the remaining resistance was attributed to the borders. Numerical modeling of water vapor in air diffusing through a bordered pit based on average dimensional data in a simplified geometry was performed by Wadsö (1988) with the finite difference method on a single bordered pit. Wadsö concluded that the resistance of the margo and torus is minor compared to the borders; for simulations of fiber-to-fiber diffusion interconnected with a high number of aspirated pits, results were similar to what was obtained by Petty (1973).

More recently, pressure-driven flow through pits has been investigated to estimate the hydraulic resistance for conduction of water in trees. This has been simulated by Valli et al. (2002) using the lattice Boltzmann method (LBM) for a single bordered pit. To reduce computational demand, the actual membrane was modeled as a porous medium. To estimate the effects of single elements, such as the margo, simulations were made with and without said elements and compared to each other to see their relative contributions. Another approach was used by Lancashire and Ennos (2002) with two analytical models: one for a single pit and one with the entire tracheid with a distribution of pits. Model results were compared with experimental data using an experimental setup for the membrane consisting of rectangular galvanized steel grids with pore sizes similar to a scaled-up membrane in a pipe setup. Although the complexity of the membrane was lost, the experiment allowed for a relative comparison of elements that cause resistance. With recent advances in computing and simulation efforts, it has been proven possible to create high-resolution models of a bordered pit, as performed by Schulte (2012) and Schulte et al. (2015), where the membrane structure is based on scanning electron microscope (SEM) images to create a realistic model of the structure.

Even though diffusion has been studied on the cellular level in wood, there, nevertheless, lacks a detailed high-resolution study and, in particular, one with relevance for larger molecules, such as high molecular weight polysaccharides and enzymes. For these macromolecules, the pit connections between cells are even more important and may be considered as bottlenecks for mass transfer. Studies have shown that steam explosion leads to ruptures in pits, which could lead to break aspiration (Zhang and Cai 2006). The effect of steam explosion on diffusion has not been studied previously.

This work aims to find an effective diffusion coefficient for larger molecules through non-aspirated bordered pits in softwood based on microscopic techniques and mathematical modeling. The effective diffusivity obtained can then be used in larger-scale simulations of tracheids to reduce computational demand, instead of resolving the smallest structures of the pits.

By using confocal laser scanning microscopy (CLSM) with the method of fluorescent recovery after photobleaching (FRAP) (Lorén et al. 2015), the diffusion coefficients were determined for larger molecules in solution. Combined with simulations in a generated geometry based on SEM images of pit membrane 
structure, the effective diffusion coefficient of the entire structure was computed. The lattice Boltzmann method (LBM) has shown great promise compared to more traditional finite difference schemes for simulations in complex geometries, such as 3D porous structures (Eshghinejadfard et al. 2016; Liu and Wu 2016; Zhang et al. 2016), due to its cost-efficient implementation of boundary conditions and ease of parallelization (Zhou 2004; Bernsdorf 2008; Gebäck et al. 2015).

Based on the simulations, the individual contribution to diffusive resistance was investigated to understand how the complex structure of a pit affects diffusion. The findings will contribute to the understanding of the effect of bordered pits on diffusion as well as give insight into pretreatment for increasing accessibility and diffusion through the porous network in softwoods.

\section{Modeling}

Two models of the pit were used in this study. The first was a high-resolution model in the LBM framework where the geometric features are based on SEM images and reported dimensional data from the literature. The goal of these simulations was to find the effective diffusion coefficient for one single bordered pit, as well as finding the resistance to diffusion of the individual structural features of the borders, margo, and torus.

The second was a diffusion in series model where the pit is divided into parts connected in series. The main goal of this model is to investigate the impact of dimensional proportions of the structural features of the pit, such as length and cross-sectional area. It is also used to compare the results of the LBM model where the structural features are resolved to investigate the importance of the path of diffusion in 3D.

Confocal microscopy was used with the FRAP technique to determine the free diffusion coefficient in a solution of dextran, while the Stokes-Einstein formulation was used to calculate the diffusion coefficient of proteins based on molecular weight. These molecules were used to represent polysaccharides present in wood and enzymes used in a biorefinery setting. The free diffusion coefficient was used to scale the results for an effective diffusion coefficient of the respective solute for the entire pit.

\section{Geometry}

The general structure and nomenclature of a bordered pit are shown in Fig. 1. Pit structure and dimension vary depending on tree species and growth conditions and, to some extent, even within the same tree. To achieve a representative value of an average bordered pit within a conifer, several different sources were used for structural data, see Table 1 . The margo, which essentially is a thin strand membrane with an impermeable torus in the center, is generally unique for each pit.

The margo was based on a scanning electron microscope (SEM) image of an earlywood bordered pit in Grand fir (Abies grandis) by Petty (1972), as shown in Fig. 2. To trace the outline of the margo and torus, the image was imported into 


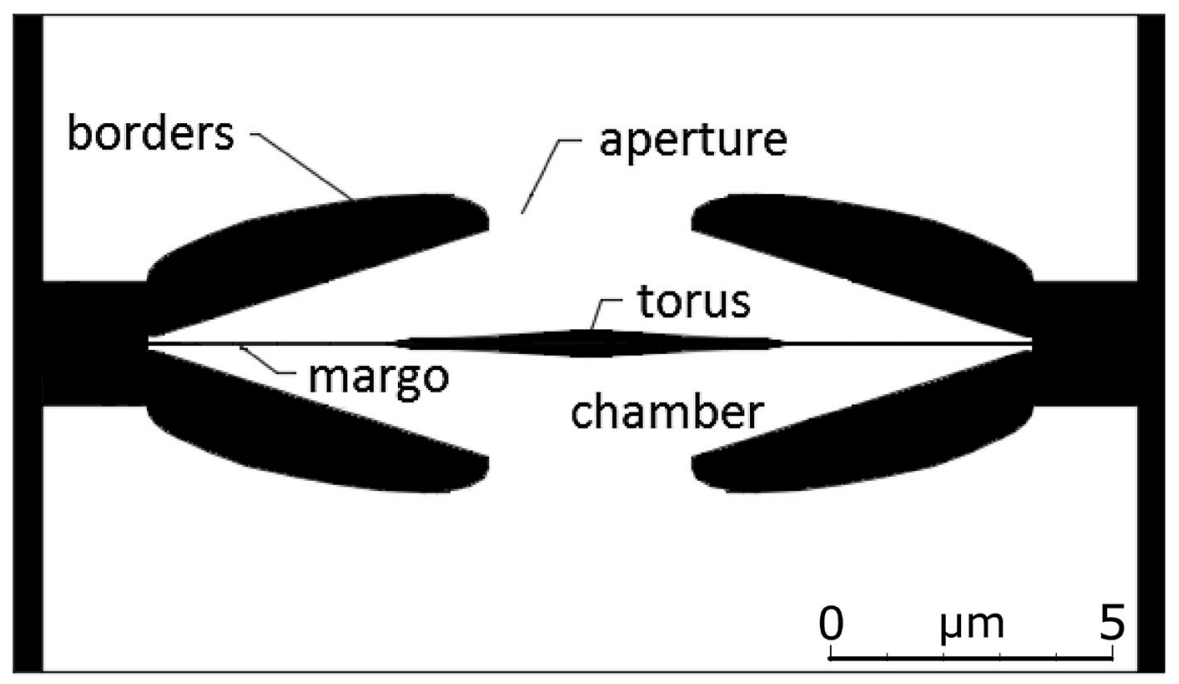

Fig. 1 General structure and nomenclature of a bordered pit with scale bar based on the values in Table 1. The borders envelop the membrane-like structure of the margo and impermeable torus in the center. The chamber is the open space within the bordered pit with the aperture leading into the pit

Table 1 Dimensions of the pit components used to generate the geometry

Based on data from Petty (1972), Siau (1984), Hacke et al. (2004), Trtik et al. 2007), Schulte (2012) and Schulte et al. (2015)

Fig. 2 To the left, vector representation of the margo extruded into three dimensions based on Petty (1972). To the right, including borders and simulations box. The borders have been partially removed for illustrative purposes

\begin{tabular}{lc}
\hline Pit component dimension & Size $(\mu \mathrm{m})$ \\
\hline Margo thickness & 0.05 \\
Torus thickness (center) & 0.50 \\
Torus diameter & 7.45 \\
Margo outer diameter & 16.10 \\
Aperture diameter & 3.70 \\
Aperture depth & 0.63 \\
Chamber depth & 1.98 \\
\hline
\end{tabular}
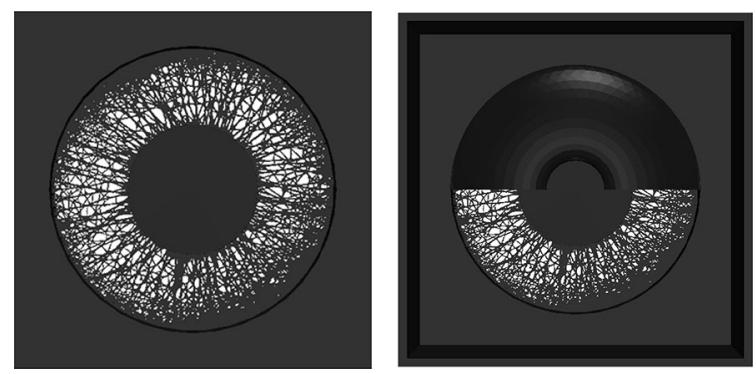

Inkscape 0.91. Using the trace bitmap command, a vectorized image was created based on pixel contrast. Further, the two-dimensional image was imported into AutoCAD 2016 where it was extruded into three dimensions. 
Additionally, the chamber and borders covering the margo were drawn up in AutoCAD with the average dimensions found in Table 1, and the full model can be seen to the right in Fig. 2. The depth, width, and height of the simulations box are 12,20 , and $20 \mu \mathrm{m}$.

To determine the resistance of each individual component of the pit, five different models/geometries were used as shown in Fig. 3. Model A represents the entire pit with borders, margo, and torus intact. Models B, C, and D are without the margo, torus, and borders, respectively. This allowed for a direct comparison to determine the relative resistance among the models and, thus, the individual resistance of the borders, margo, and torus.

To simulate the effect that steam explosion may have on the structure of the pit, Model E was developed. In that model, it is assumed that the treatment has opened or ruptured the borders and that the margo and torus have been removed.

\section{Lattice Boltzmann simulations}

The lattice Boltzmann method was used to solve Fick's second law of diffusion in the pit structure, i.e.,

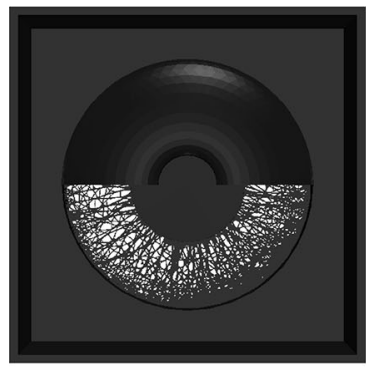

Model A

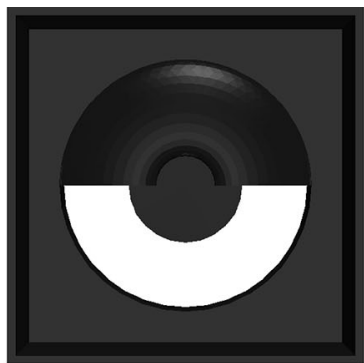

Model B

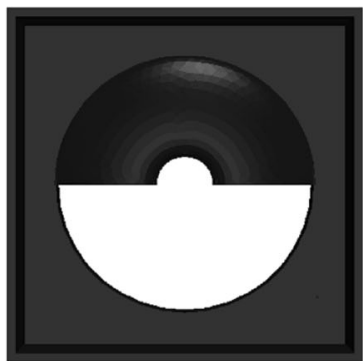

Model C

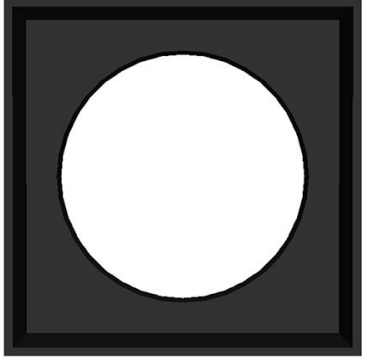

Model D

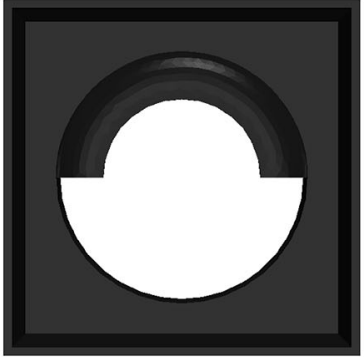

Model E

Fig. 3 Different models used in the LBM simulations. Borders have been partially removed for illustrative purposes. Model $A$ is the full model with borders, margo, and torus. Models $B-D$ have had the margo, torus, and borders removed to assess individual resistances relative to Model A. Model E represents a pit that has undergone steam explosion in which parts of the structure have been removed and the borders have detached somewhat 


$$
\frac{\partial C}{\partial t}=D_{0} \nabla^{2} C
$$

where $C$ is the concentration and $D_{0}$ is the free diffusion coefficient in the open space. Cell walls, borders, margo, and torus were treated as impermeable walls with a zero normal flux boundary condition:

$$
-D_{0} \frac{\partial C}{\partial n}=0
$$

A constant concentration difference was applied between the inlet and outlet of the pit to create a concentration gradient. By solving the diffusion equation to steady state, the effective diffusion coefficient $D_{\text {eff }}$ was obtained from the average flux from Fick's first law of diffusion according to

$$
\overline{J_{x}}=-D_{\text {eff }} \frac{\Delta C}{\Delta x}
$$

where $\Delta x$ is the thickness of the simulation box, $\Delta C$ is the applied concentration difference and $\overline{J_{x}}$ is the mean flux.

The simulation box was computed on a uniform grid of $500 \times 500 \times 286$ voxels, and the LBM used a two-relaxation-time method for diffusion (Ginzburg 2005) which benefits from improved stability while being equal in terms of both computational time and simplicity compared to the classical BGK approximation. A D3Q19 setup was used where the boundary conditions in Eq. 2 were realized using a ghost node scheme according to Gebäck and Heintz (2014).

The ghost node scheme utilizes a mirror point inside the domain where the macroscopic variables are found by interpolation, subsequently used to obtain the correct boundary condition by assigning an appropriate distribution function on the ghost node. All streaming directions of the ghost node are not necessary to implement the boundary condition, which makes it possible to implement the boundary condition even in the case of a thin boundary with the width of a single ghost node.

\section{Resistance to diffusion}

To assess the contribution of individual components to the resistance to diffusion through a bordered pit, the effective diffusion coefficient for each model (A, B, and C) was related to Model D, as shown in Eq. 4:

$$
z_{i}=1-\frac{D_{\mathrm{eff}, i}}{D_{\mathrm{eff}, D}}
$$

where $z_{i}$ is the normalized decrease in the diffusion coefficient caused by the obstacles imposed by Model $i$, compared to if the pit was solely a circular opening in the cell wall. Thus, the resistance of the different components can be expressed by comparing the normalized decrease to Model A, as in Eqs. 5, 6, and 7. Note that the total sum of resistances is equal to 1 . 


$$
\begin{gathered}
R_{\text {borders }}=\frac{z_{\mathrm{C}}}{z_{\mathrm{A}}} \\
R_{\text {torus }}=\frac{z_{\mathrm{B}}-z_{\mathrm{C}}}{z_{\mathrm{A}}} \\
R_{\text {margo }}=\frac{z_{\mathrm{A}}-z_{\mathrm{B}}}{z_{\mathrm{A}}}
\end{gathered}
$$

\section{Simplified diffusion in series model}

Rewriting Eq. 3, replacing the mean flux with the molar flow rate divided by the area, an expression for the molar flow rate through the bordered pit is obtained as:

$$
n=-D_{\text {eff }} A \frac{\Delta C}{\Delta x}
$$

where $n$ is the total molar flow rate of the diffusing species, $D_{\text {eff }}$ is the effective diffusion coefficient, and $\mathrm{A}$ is the cross-sectional area of the entire pit. To describe the molar flow rate in each section of the pit, it was assumed that free diffusion is prevalent in the open parts of the pit, i.e.,

$$
n_{i}=-D_{0} A_{i} \frac{\Delta C_{i}}{\Delta x_{i}}
$$

where $i$ represents one of the sections and $A_{i}$ is the area of that section. To solve for the effective diffusion coefficient, the individual molar flow rates $n_{i}$ must be equal to the total flow rate $n$, and thus, Eq. 10 is obtained, in which the whole pit is divided into 2 apertures, 2 chambers, and 1 membrane.

$$
D_{\mathrm{eff}}=\frac{D_{0} \Delta x}{A\left(2 \frac{\Delta x_{a}}{A_{a}}+2 \frac{\Delta x_{c}}{A_{c}}+\frac{\Delta x_{m}}{A_{m}}\right)}
$$

Similarly to the resistance to diffusion in the LBM model, individual mass transfer resistances for the simple model can be expressed as:

$$
\begin{aligned}
R_{\text {Borders }} & =\frac{2 \frac{\Delta x_{a}}{A_{a}}+2 \frac{\Delta x_{c}}{A_{c}}}{2 \frac{\Delta x_{a}}{A_{a}}+2 \frac{\Delta x_{c}}{A_{c}}+\frac{\Delta x_{m}}{A_{m}}} \\
R_{\text {Membrane }} & =\frac{\frac{\Delta x_{m}}{A_{m}}}{2 \frac{\Delta x_{a}}{A_{a}}+2 \frac{\Delta x_{c}}{A_{c}}+\frac{\Delta x_{m}}{A_{m}}}
\end{aligned}
$$

Table 2 shows the cross-sectional areas used for the above equations based on the geometry of Model A. 
Table 2 Cross-sectional area used in the simplified model

\begin{tabular}{lcl}
\hline Sections & Area $\left(\mu \mathrm{m}^{2}\right)$ & Length $(\mu \mathrm{m})$ \\
\hline Aperture & 10.75 & 0.63 \\
Chamber & 107.2 & 1.98 \\
Membrane (margo + torus) & 40.49 & 0.05 \\
Entire pit & 203.6 & 5.27 \\
\hline
\end{tabular}

Note that the margo and torus were assumed to be of equal thickness and combined into one section called the membrane. The total area was calculated from the outer diameter of the margo, similar to Model D

\section{Diffusion coefficients in free solution}

Fluorescence recovery after photobleaching (FRAP) is an established technique (Lorén et al. 2015) used to obtain information on the diffusion dynamics of fluorescent molecules commonly used with confocal microscopes. Labeled fluorescent probes in solution are irreversibly bleached in a volume by high intensity light, which causes a concentration gradient within the volume. Recovery of the fluorescent signal in this area will be a function of the ability of unbleached probes to diffuse into said region of interest.

FRAP measurements were taken on a Leica SP5 confocal microscope AOBS (Heidelberg, Germany) with a $20 \times, 0.5 \mathrm{NA}$ water immersion objective. The following settings were used: $256 \times 256$ pixels; zoom factor 4 (with a zoom-in during bleaching); and $1000 \mathrm{~Hz}$, rendering an acquisition rate of $0.265 \mathrm{~s}$ per image and a pixel size of $0.73 \mu \mathrm{m}$. Beam expander 1 was used, which lowered the effective NA to approximately 0.35 and yielded a slightly more cylindrical bleaching profile. The FRAP images were stored as 12-bit TIFF images. The FRAP protocol consisted of 20 prebleach images, 1-4 bleach images (in order to achieve an initial bleaching depth of $30 \%$ of the prebleach intensity) followed by 50 images obtained during the recovery process.

The respective fluorescent diffusion probes FITC-Dextran 3, 10, and $40 \mathrm{kDa}$ (Invitrogen Molecular Probes, Eugene, OR) were dispersed in distilled water to yield $200 \mathrm{ppm}$. The solutions were placed on a cover glass slip in a well created by Secure-Seal ${ }^{\mathrm{TM}}$ Spacers (Thermo Fisher Scientific, Sweden), and a second cover glass slip was used to seal the well. The FRAP model called "Maximum likelihood estimation for FRAP data with a Gaussian starting profile" (Jonasson et al. 2008) was used to evaluate the data in MATLAB (MathWorks, USA).

The Stokes-Einstein equation (Eq. 13) can be used to estimate the diffusion coefficients of large rigid spherical particles in dilute liquids, as well as calculate the radius of the diffusing species based on the diffusion coefficient (Cussler 2009).

$$
D_{0}=\frac{k_{\mathrm{B}} T}{6 \pi \mu R}
$$

Here, $k_{\mathrm{B}}$ is the Boltzmann's constant, $T$ is the temperature, $\mu$ is the viscosity of the solvent, and $R$ is the radius of the diffusing molecule. Dissolved proteins commonly 
form spherical globules (Erickson 2009), which enable an estimate to be performed based on Eq. 13. To calculate the radius of an assumed spherical protein of a given mass, Eq. 14 is used:

$$
R=0.066 M_{\mathrm{w}}^{1 / 3}
$$

where $M_{\mathrm{w}}$ is the molecular weight in Dalton and $R$ is the radius in nanometers of the protein. Caution should be taken as this is the minimum radius for a smooth sphere, while in reality the average radius will be larger than this (Erickson 2009). In this study, the span of molecular weights under consideration was based on commonly used enzymes in the biorefinery area, which are in the range of $40-80 \mathrm{kDa}$.

\section{Results and discussion}

The free diffusion coefficients in an aqueous solution are listed in Table 3. As should be expected, the larger species are much slower than the smaller ones, and since dextran is a long polymer chain, it will differ significantly from the traditional spherical assumption of solutes. An indication of this can clearly be seen from comparing the $40 \mathrm{kDa}$ dextran with the $40 \mathrm{kDa}$ protein. The smallest probe was in the higher range of what can be measured reliably with the FRAP technique (Lorén et al. 2015), which was reflected in the standard deviation. Previous studies by Arrio-Dupont et al. (1996) on dextran probes agree well with the results here.

The effective diffusion coefficients calculated for the different models of the pits using the LBM are presented in Figs. 4 and 5. The results have been scaled to exclude the simulation box such that the effective diffusion coefficient represents only the bordered pit.

Three different results are shown based on which diffusion probe was used to scale the result. The difference in coefficients between Models A and B is very small, which indicates that the margo did not hinder diffusion much at all. Model $\mathrm{C}$ shows a small increase because the torus was not present, and the path of diffusion was shorter. Without the borders present in Model D, a large increase in the

Table 3 Diffusion coefficients in an aqueous solution

\begin{tabular}{lrll}
\hline Diffusion probe & Molecular weight $(\mathrm{kDa})$ & Diffusion coefficient $\left(\mu \mathrm{m}^{2} / \mathrm{s}\right)$ & Hydrodynamic radius $(\mathrm{nm})$ \\
\hline FITC-Dextran & 3 & $147.6 \pm 10.1$ & $1.66 \pm 0.10$ \\
& 10 & $65.0 \pm 2.3$ & $3.77 \pm 0.13$ \\
& 40 & $41.4 \pm 0.6$ & $5.92 \pm 0.08$ \\
Protein & 40 & 109 & 2.26 \\
& 60 & 95 & 2.58 \\
& 80 & 86 & 2.84 \\
\hline
\end{tabular}

Dextran diffusion probes were measured by confocal microscopy with the FRAP technique, while the proteins are calculated using Eq. 13. The equivalent radius in solution is presented as a comparison among the species 


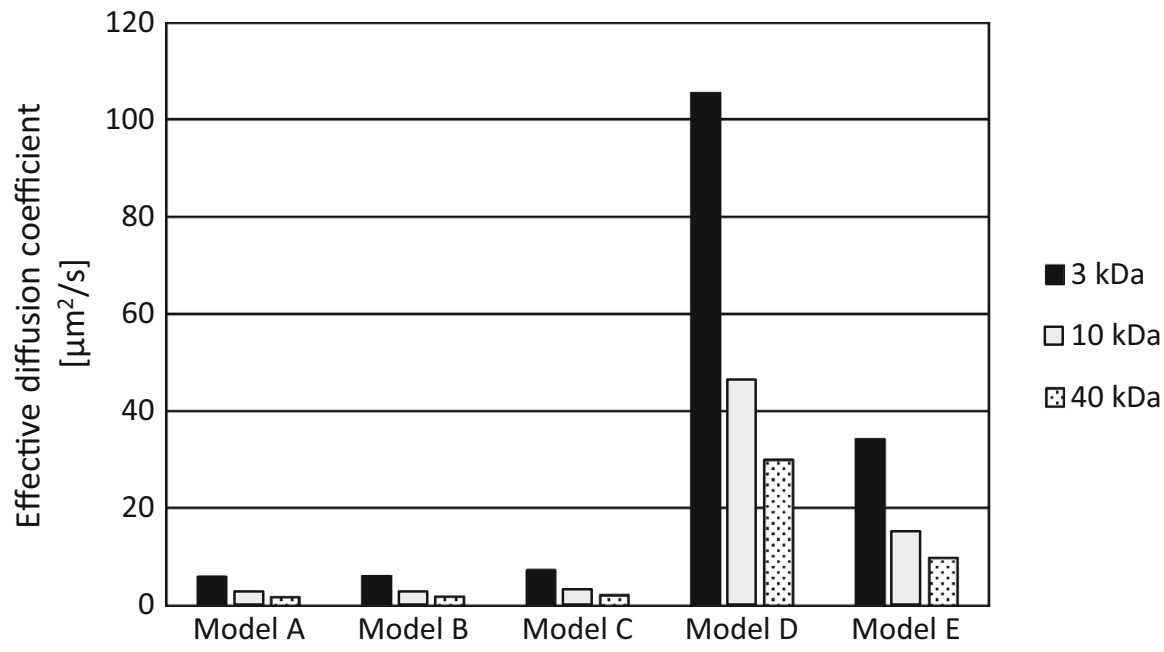

Fig. 4 Effective diffusion coefficients using the five LBM models and the dextran diffusion probes of 3, 10 , and $40 \mathrm{kDa}$. The effective diffusion coefficient is given in $\mu \mathrm{m}^{2} / \mathrm{s}$

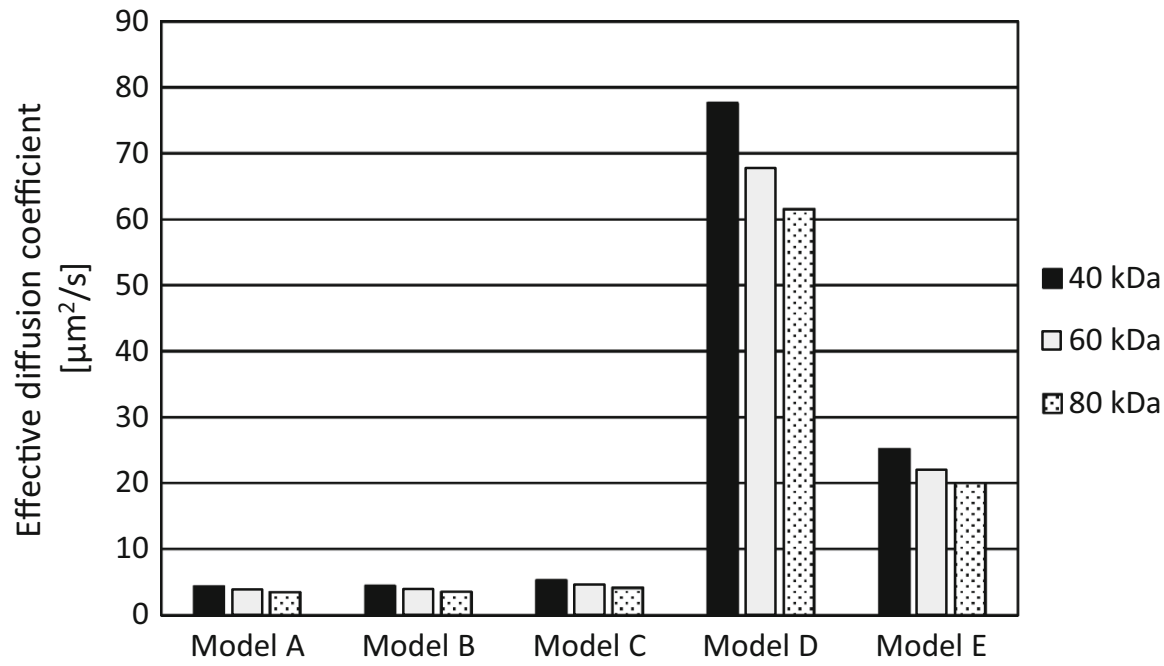

Fig. 5 Effective diffusion coefficients using the five LBM models and the calculated values for the proteins of 40,60, and $80 \mathrm{kDa}$. The effective diffusion coefficient is given in $\mu \mathrm{m}^{2} / \mathrm{s}$

diffusion coefficient can be seen due to the large increase in the cross-sectional area. While in Model $\mathrm{E}$ where the borders have been deformed due to steam explosion, but are still present, the increase is not as large as in Model A but still significantly higher than Models $\mathrm{A}, \mathrm{B}$, and $\mathrm{C}$.

To illustrate the path of diffusion for Model A, integrated flux lines from the LBM simulation are shown in Fig. 6 for a cross-sectional slice. The flux lines curve 


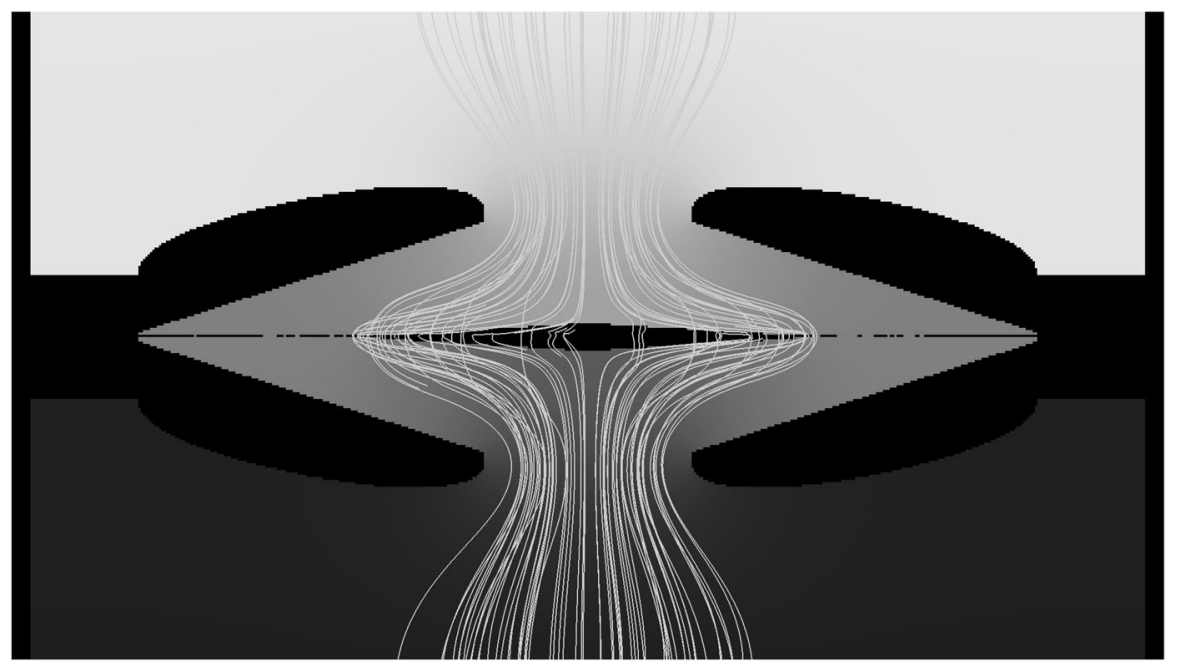

Fig. 6 A 2D cross-sectional slice of the simulations for Model A. The solid walls are seen in black, while the concentration gradient is in gray scale where high concentration is darker and low concentration lighter. Integrated lines of the flux field are made visible through the structure based on a point source generated with the stream tracer filter in ParaView

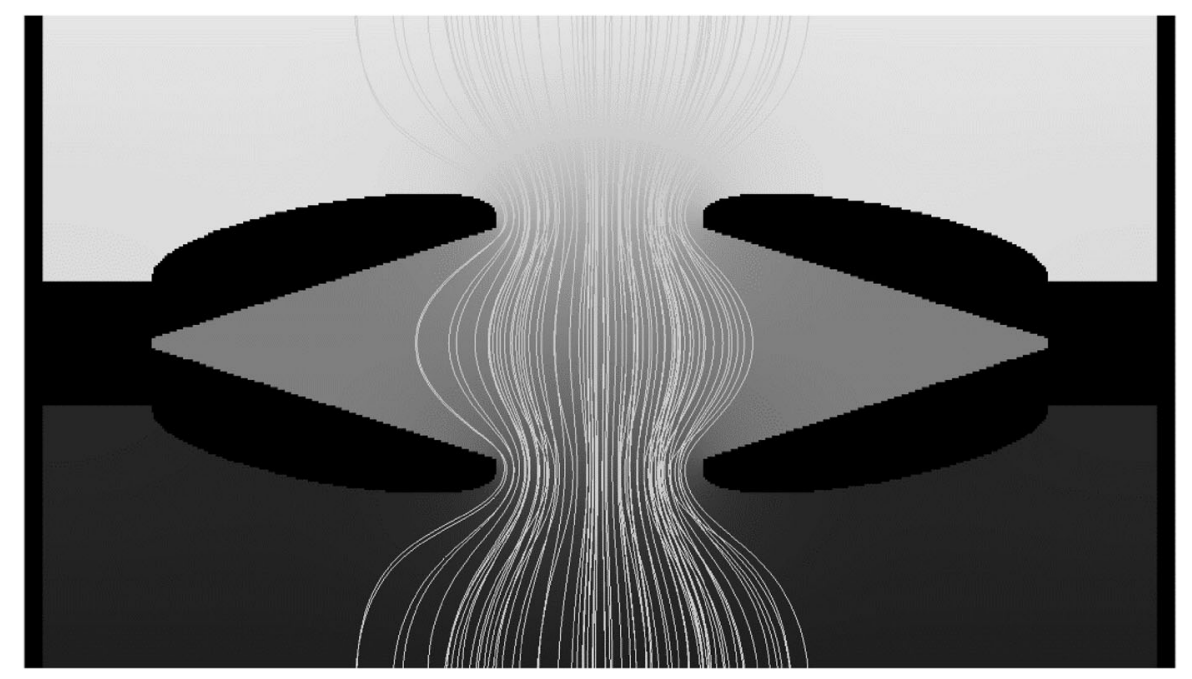

Fig. 7 A 2D cross-sectional slice of the simulations for Model C. The solid walls are seen in black, while the concentration gradient is in gray scale where high concentration is darker and low concentration lighter. Integrated lines of the flux field are made visible through the structure based on a point source generated with the stream tracer filter in ParaView

around the torus close to the surface, whereas in Fig. 7 for Model C, without torus or margo present, the lines have a more straight path. Models D and E are similar to Fig. 7 but with a larger diameter of the aperture leading into the chamber of the pit, 
and the lines have less curvature. This also illustrates the difference between resolving the full 3D structure as compared to the simple model in only 1D.

Based on the simulations for Models $\mathrm{A}$ and $\mathrm{E}$, equations for the effective diffusion coefficient can be expressed for the entire undisturbed pit and the steamexploded pit with the following equations as a function of the free diffusion coefficient:

$$
\begin{gathered}
D_{\text {eff }}=0.040 D_{0} \\
D_{\text {eff,Stex }}=0.232 D_{0}
\end{gathered}
$$

The effective diffusion coefficient from the simplified model is four times as large as the effective diffusion coefficient from the LBM simulations, as shown in Fig. 8. This is reasonable since the simplified expression only considers the crosssectional areas available for diffusion across a pit and not the tortuous path between the pit components, illustrated by the flux lines in Figs. 6 and 7.

By using Eqs. 5, 6, and 7, it was found that the borders constitute most of the resistance to diffusion and that the resistances imposed by the torus and margo are negligible in comparison. The border and membrane resistances from the simplified diffusion model using Eqs. 11 and 12 present a very similar result pointing out the borders as the main resistance to diffusion, as listed in Table 4.

The large border resistance is reasonable since the cross-sectional area available for diffusion in the apertures is small compared to the cross-sectional areas for the other components and since the torus and margo are very thin compared to the rest of the pit.

To ensure that the grid size was sufficient to resolve the membrane structure in Model A, a grid refinement study was performed. The change in the solution

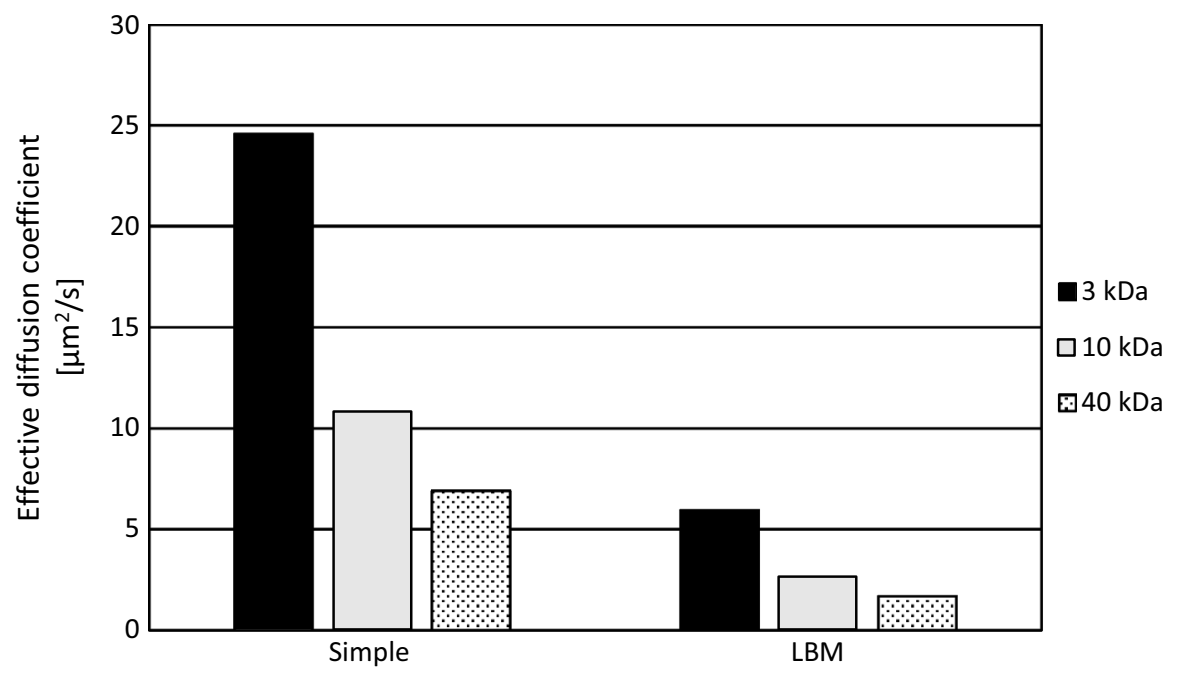

Fig. 8 Effective diffusion coefficient for the simplified model with diffusion in series compared to the more complex LBM simulations. Results shown are for dextran 3, 10, and $40 \mathrm{kDa}$ 
Table 4 Fractional diffusive resistance of the borders, torus, and margo for the LBM model and the simplified model

\begin{tabular}{lcc}
\hline Sections & Resistance LBM $(\%)$ & Resistance simple (\%) \\
\hline Borders & 98.0 & 99.2 \\
Torus & 1.8 & 0.8 \\
Margo & 0.2 & \\
\hline
\end{tabular}

For the simple model, the torus and margo were merged into one membrane section

obtained was seen to be less than $0.5 \%$ with additional refinement. To additionally confirm that the smallest channels in the margo were resolved and an accurate solution was obtained, grid refinement was performed on a model with only the margo and torus present. The geometry was further scaled, while the simulation box was kept constant to effectively double the resolution. Results obtained from this revealed a very minor decrease in the effective diffusion coefficient. The dominating part of the resistance is clearly located at the borders, and this minor decrease would not significantly alter the result.

To investigate the significance of the membrane further, the membrane thickness and area were varied in the simplified model. An increase in membrane thickness from 50 to $500 \mathrm{~nm}$ resulted in a $6.7 \%$ decrease in the effective diffusion coefficient, while a decrease in thickness to $5 \mathrm{~nm}$ was insignificant. For a thickness of $500 \mathrm{~nm}$, the contribution of membrane to resistance increased to $7.4 \%$, still very low compared to the borders. A 20 -fold decrease in the cross-sectional membrane area resulted in a $13.1 \%$ decrease in the diffusion coefficient and a $13.8 \%$ increase in the contribution to resistance. Further decrease in the area of the membrane will increase the resistance; however, the membrane is still limited by its thickness compared to the entire pit. The path of diffusion also starts to change as the area decreases, thus increasing tortuosity and giving increasingly misleading values.

Schulte (2012) and Schulte et al. (2015) modeled water flow through bordered pits and reported that the margo and torus together constitute more than $80 \%$ of the flow resistance. Valli et al. (2002) ascribed 38\% of the flow resistance to the margo. The membrane resistance for diffusion was found to be close to $2 \%$ in the present study. The large difference is explained by the presence of shear stresses in flow and the large surface area of the thin stranded membrane structure of the margo, while for diffusion the available cross-sectional area and length of the margo pores control the resistance. Note that for diffusion, the presence of a structure does not influence the tangential flux along the structure, while for flow, the no-slip boundary condition imposes zero tangential velocity at the structure boundary, which has a large effect on the flow resistance, especially in narrow channels.

Model E represents a pit in which steam explosion has removed all components such that only an opening in the cell wall remains. The resistance to diffusion markedly decreases if the border diameter is affected and that alone will increase the effective diffusion coefficient by a factor 4, as shown in Figs. 4 and 5. If the diameter is further decreased, it eventually reaches the same result as in the Model $\mathrm{D}$ and illustrates the importance of the borders in the case of diffusion through a bordered pit. 


\section{Conclusion}

This study determined the effective diffusion coefficient of large molecules in a conifer earlywood bordered pit by using the lattice Boltzmann method and confocal microscopy. The effective diffusivity obtained can be used in larger-scale simulations of tracheids to reduce computational demand without resolving the smallest structures of the pits.

The individual resistance of structural features to diffusion was investigated. Contrary to what is found for pressure-driven flow in which the margo and torus contribute to the major part of flow resistance, it was found that the borders totally dominate mass transfer resistance for diffusion. Simulations of an opened pit structure (mimicking steam explosion) gave a significant enhancement of mass transfer.

Two estimates for the effective diffusion coefficient $D_{\text {eff }}$ (Eqs. 15 and 16) were presented for an undisturbed pit and a steam-exploded pit based on the free diffusion coefficient $D_{0}$ in the solution. With this result and a suitable way of finding $D_{0}$ for a solute, the $D_{\text {eff }}$ of the entire pit for a specific solute can be estimated.

Acknowledgement The authors would like to thank Wallenberg Wood Science Center for financial support and SuMo Biomaterials for support with confocal microscopy and simulations.

Open Access This article is distributed under the terms of the Creative Commons Attribution 4.0 International License (http://creativecommons.org/licenses/by/4.0/), which permits unrestricted use, distribution, and reproduction in any medium, provided you give appropriate credit to the original author(s) and the source, provide a link to the Creative Commons license, and indicate if changes were made.

\section{References}

Alvira P, Tomás-Pejó E, Ballesteros M, Negro MJ (2010) Pretreatment technologies for an efficient bioethanol production process based on enzymatic hydrolysis: a review. Bioresour Technol 101:4851-4861

Arrio-Dupont M, Cribier S, Foucault G, Devaux PF, d‘Albis A (1996) Diffusion of fluorescently labeled macromolecules in cultured muscle cells. Biophys J 70:2327-2332. doi:10.1016/S00063495(96)79798-9

Asikainen A (2010) Availability of wood biomass for biorefining. Cellul Chem Technol 44:111-115

Azhar S, Wang Y, Lawoko M, Henriksson G, Lindström ME (2011) Extraction of polymers from enzyme-treated softwood. BioResources 6:4606-4614

Bernsdorf J (2008) Simulation of complex flows and multi-physics with the Lattice-Boltzmann method. Dissertation, University of Amsterdam

Cussler EL (2009) Diffusion, mass transfer in fluid systems, 3rd edn. Cambridge University Press, Cambridge

Erickson HP (2009) Size and shape of protein molecules at the nanometer level determined by sedimentation, gel filtration, and electron microscopy. Biol Proced Online 11:32-51

Eshghinejadfard A, Daróczy L, Janiga G, Thévenin D (2016) Calculation of the permeability in porous media using the lattice Boltzmann method. Int J Heat Fluid Flow 1329:93-103

FitzPatrick M, Champagne P, Cunningham MF, Whitney RA (2010) A biorefinery processing perspective: treatment of lignocellulosic materials for the production of value-added products. Bioresour Technol 101:8915-8922 
Galbe M, Zacchi G (2002) A review of the production of ethanol from softwood. Appl Microbiol Biotechnol 59:618-628

Gebäck T, Heintz A (2014) A lattice Boltzmann method for the advection-diffusion equation with Neumann boundary conditions. Commun Comput Phys 15:487-505

Gebäck T, Marucci M, Boissier C, Arnehed J, Heintz A (2015) Investigation of the effect of the tortuous pore structure on water diffusion through a polymer film using Lattice Boltzmann simulations. J Phys Chem B 119:5220-5227

Ginzburg I (2005) Equilibrium-type and link-type lattice Boltzmann models for generic advection and anisotropic-dispersion equation. Adv Water Resour 28:1171-1195

Hacke UG, Sperry JS, Pitterman J (2004) Analysis of circular bordered pit function II. Gymnosperm tracheids with torus-margo pit membranes. Am J Bot 91:386-400

IPCC (2007) Climate change 2007: synthesis report. Contribution of Working Groups I, II and II of the fourth assessment report of the Intergovernmental Panel on Climate Change. Geneva, Switzerland

Jedvert K, Wang Y, Saltberg A, Henriksson G, Lindström ME, Theliander H (2012) Mild steam explosion: a way to activate wood for enzymatic treatment, chemical pulping and biorefinery processes. Nord Pulp Pap Res J 27:828-835

Jonasson JK, Loren N, Olofsson P, Nyden M, Rudemo M (2008) A pixel-based likelihood framework for analysis of fluorescence recovery after photobleaching data. J Microsc 232:260-269

Lancashire JR, Ennos AR (2002) Modelling the hydrodynamic resistance of bordered pits. J Exp Bot 53:1485-1493

Liu Z, Wu H (2016) Pore-scale study on flow and heat transfer in 3D reconstructed porous media using micro-tomography images. Appl Therm Eng 100:602-610

Lorén N, Hagman J, Jonasson JK et al (2015) Fluorescence recovery after photobleaching in material and life sciences: putting theory into practice. Q Rev Biophys 48:323-387

Ma Q, Zhao Z, Xu M, Yi S, Wang T (2016) The pit membrane changes of micro-explosion-pretreated poplar. Wood Sci Technol 50:1089-1099

Muzamal M, Gamstedt EK, Rasmuson A (2014) Modeling wood fiber deformation caused by vapor expansion during steam explosion of wood. Wood Sci Technol 48:353-372

Muzamal M, Jedvert K, Theliander H, Rasmuson A (2015) Structural changes in spruce wood during different steps of steam explosion pretreatment. Holzforschung 69:61-66

Muzamal M, Bååth Arnling J, Olsson L, Rasmuson A (2016) Contribution of structural modification to enhanced enzymatic hydrolysis and 3-D structural analysis of steam-exploded wood using X-ray tomography. BioResources 11:8509-8521

Petty JA (1972) The aspiration of bordered wood pits in conifer wood. Proc R Soc London 181:395-406

Petty JA (1973) Diffusion of non-swelling gases through dry conifer wood. Wood Sci Technol 7:297-307

Schulte PJ (2012) Computational fluid dynamics models of conifer bordered pits show how pit structure affects flow. New Phytol 193:721-729

Schulte PJ, Hacke UG, Schoonmaker AL (2015) Pit membrane structure is highly variable and accounts for a major resistance to water flow through tracheid pits in stems and roots of two boreal conifer species. New Phytol 208:102-113

Siau JF (1984) Transport processes in wood. Springer, Berlin

Sjöström E (1993) Wood chemistry: fundamentals and applications, 2nd edn. Academic Press Inc, San Diego

Stamm A (1946) Passage of liquids, vapors, and dissolved materials through softwoods. Tech Bull - US Dept Agric 84

Trtik P, Dual J, Keunecke D et al (2007) 3D imaging of microstructure of spruce wood. J Struct Biol 159:46-55

Valli A, Koponen A, Vesala T, Timonen J (2002) Simulations of water flow through bordered pits of conifer xylem. J Stat Phys 107:1-3

Wadsö L (1988) Bordered pit diffusion, report TVBM-3034. Lund Institute of Technology, Sweden

Zhang Y, Cai L (2006) Effects of steam explosion on wood appearance and structure of sub-alpine fir. Wood Sci Technol 40:427-436

Zhang X, Crawford JW, Young IM (2016) A Lattice Boltzmann model for simulating water flow at pore scale in unsaturated soils. J Hydrol 538:152-160

Zhou JG (2004) Lattice Boltzmann methods for shallow water flows. Springer, Berlin 